\title{
Producción de recubrimientos de fosfato cálcico mediante ablación láser para aplicaciones biomédicas
}

\author{
M.B. Mayor ${ }^{(*)}$, J.L. Arias ${ }^{(*)}$, F.J. García-Sanz ${ }^{(*)}$, J. Pou $^{(*)}$, B. León ${ }^{(*)}$ y M. Pérez-Amor ${ }^{(*)}$
}

Resumen Los fosfatos cálcicos apatíticos se utilizan como recubrimientos para mejorar la fijación de implantes ortopédicos y dentales. La técnica comercial para la producción de estos recubrimientos (plasmaspray) presenta problemas de adherencia y control de propiedades físico-químicas. En esta comunicación, se presenta la ablación láser como alternativa para solventar estos problemas. El sistema de ablación consiste en una cámara de vacío llena de vapor de agua hasta la presión deseada. El haz de un láser excímero de ArF se focaliza en un blanco de hidroxiapatito, depositándose el recubrimiento en un substrato paralelo al blanco mantenido a una cierta temperatura. Las propiedades físico-químicas del material se analizaron por espectroscopía infrarroja por transformada de Fourier (FT-IR), difracción de rayos X (XRD), microscopía electrónica de barrido (SEM) y espectroscopía de rayos X por dispersión de energías (EDX). Basándose en estos resultados, se escogieron dos tipos de recubrimientos para determinar su biocompatibilidad in vitro.

Palabras clave: Ablación láser. Recubrimiento. Fosfato cálcico. Implante.

\section{Production of calcium phosphate coatings by laser ablation for biomedical applications}

\begin{abstract}
Apatitic calcium phosphates are used as coatings to improve the fixation of orthopaedic and dental implants. The commercial technique to produce these coatings (plasm-spray) shows some problems of adherence and control of their physicochemical properties. In this communication laser ablation is presented as a solution to overcome these problems. The ablation system consists in a vacuum chamber filled with water vapour up to the desired pressure. The laser beam of an ArF excimer laser is focused on a hydroxyapatite target, and the coating is deposited on a substrate parallel to the target maintained at certain temperature. The physicochemical properties of the material were analysed by Fourier transformed infrared spectroscopy (FT-IR) $X$ ray diffraction (XRD), scanning electron microscopy (SEM), and energy dispersive $X$ ray spectroscopy (EDX). Taking into account these results, two types of coatings were choosen to asses their biocompatibility in vitro.
\end{abstract}

Keywords: Laser ablation. Coating. Calcium phosphate. Implant.

\section{INTRODUCCIÓN}

Las cerámicas de fosfato cálcico tipo apatito desempeñan un papel muy importante como biomateriales para aplicaciones tanto dentales como ortopédicas. Entre ellas, el hidroxilapatito (HA), $\mathrm{Ca}_{10}\left(\mathrm{PO}_{4}\right)_{6}(\mathrm{OH})_{2}$, está siendo extensivamente estudiado debido a su similitud con la parte inorgánica del hueso (1). En sí, el HA es quebradizo y relativa-

(*) Dpto. de Física Aplicada. E.T.S.I.I.M. Univ. de Vigo, Lagoas-Marcosende 9. 36200-Vigo (España). mente débil, con lo cual no puede utilizarse para aplicaciones que necesiten soportar grandes solicitaciones mecánicas (2). Por lo tanto, el HA se suele utilizar cómo recubrimiento para mejorar la fijación y osteointegración de implantes de titanio sin cementar (3).

La técnica empleada comúnmente para producir estos recubrimientos es el plasma-spray. Los recubrimientos obtenidos por este método, aunque exhiben una buena biocompatibilidad, presentan algunos inconvenientes, entre los cuales los más importantes son la pobre adherencia recubrimiento-substrato (haciendo necesario un 
tratamiento superficial de granallado, para garantizar un mínimo de adherencia) y la falta de uniformidad del recubrimiento desde dos puntos de vista diferentes: morfología y cristalinidad (4 y 5).

Debido a estas desventajas, otras técnicas han sido objeto de estudio durante esta última década, tales como la deposición electroforética, deposición por magnetron sputtering o deposicion por sputtering de haz de iones, pero sus resultados no han sido todo lo esperanzadores que debieran para que se hayan implementado de forma industrial.

Últimamente, la ablación láser se ha aplicado con éxito para la producción de recubrimientos de fosfato cálcico de diferente composición, estructura y cristalinidad (9-12). A este respecto, la técnica de ablación láser parece ser capaz de resolver los problemas intrínsecos del método de plasma-spray.

El propósito de este estudio es mostrar las posibilidades de un recubrimiento de fosfato cálcico, así como su biocompatibilidad.

\section{PARTE EXPERIMENTAL}

\subsection{Material precursor}

Los blancos utilizados para la ablación eran discos producidos a partir de polvo de HA presionado a $82 \mathrm{MPa}$ y luego sinterizado a $1.260^{\circ} \mathrm{C}$. El análisis de estos blancos por difracción de rayos X (XRD) y espectroscopía infrarroja por transformada de Fourier (FT-IR) mostraron que los blancos eran HA con substitución parcial de carbonato $\left(\mathrm{CO}_{3}^{2}\right)$ en la posición del fosfato $\left(\mathrm{PO}_{4}^{3}\right)$ y un bajo contenido en grupos hidroxilo. Medidas por fluorescencia de rayos $\mathrm{X}(\mathrm{XRF})$ de los blancos determinaron que su relación calcio/fósforo $(\mathrm{Ca} / \mathrm{P})$ era de 1,7 con una impureza de magnesio del $0,27 \%$ en peso.

\subsection{Producción de recubrimientos}

El sistema de ablación consiste en una cámara de procesamiento, en la que se efectuó un vacío base de $10^{-6}$ mbar, y luego se llenó con vapor de agua hasta la presión de procesamiento deseada. El láser (láser de excímero de $\mathrm{ArF},=193 \mathrm{~nm}$ ) se focalizó en el blanco de HA, alcanzando una densidad de energía de $0,8 \mathrm{Jcm}^{-2}$. El substrato de titanio se colocó enfrente del blanco, en un soporte de temperatura controlada (13). El tiempo de deposición se escogió de modo que el espesor de los recubrimientos fuera de $1 \mu \mathrm{m}$.

\subsection{Caracterización de los recubrimientos}

Se han utilizado diferentes técnicas para caracterizar las muestras recubiertas, incluyendo espec- troscopía por transformada de Fourier (FT-IR), difracción de rayos $\mathrm{X}$ (XRD), microscopía electrónica de barrido (SEM) y análisis químico por energías de rayos $\mathrm{X}$ dispersados (EDX). Además, las técnicas de elipsometría y perfilometría se han empleado para evaluar el espesor de los recubrimientos. La adhesión entre recubrimiento y substrato se determinó midiendo su resistencia a la tracción, siguiendo el procedimiento ASTM C633 modificado.

\subsection{Pruebas biológicas}

Se seleccionaron dos tipos diferentes de recubrimientos para determinar su biocompatibilidad in vitro mediante el estudio del anclaje y proliferación sobre ellos de células de tipo osteoblasto MG63. Con fines de comparación, también se incluyeron muestras recubiertas por plasma-spray y titanio sin recubrir, usando como controles de citotoxidad positivo solución phenol al $0,64 \mathrm{mg} / \mathrm{ml}$ y negativo poliestireno tratado. Las células se cultivaron durante tiempos comprendidos entre 0,5 horas y 18 días.

\section{RESULTADOS Y DISCUSIÓN}

La figura 1 muestra los espectros de XRD de una serie de recubrimientos de fosfato cálcico depositados en titanio a 0,45 mbar de vapor de agua y variando la temperatura del substrato entre $300 \mathrm{y}$ $525^{\circ} \mathrm{C}$.

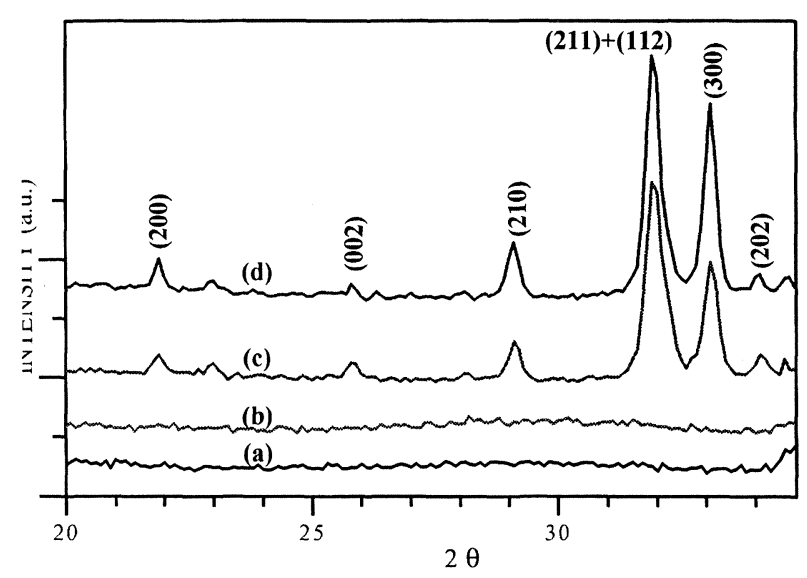

FIG. 1.- Espectros de XRD de los recubrimientos producidos por ablación láser a una presión de vapor de agua de 0,45 mbar y a diferentes temperaturas del substrato: a) $300, b$ ) $430, c) 485$, y d) $525^{\circ} \mathrm{C}$.

FIG. 1.- XRD spectra of the coatings produced by laser ablation at 0.45 mbar of water vapour pressure and at different substrate temperatures: a) 300 , b) 430 , c) 485 , and d) $525^{\circ} \mathrm{C}$. 
Como puede observarse, las temperaturas más bajas conducen a un material amorfo, mientras que los recubrimientos producidos a temperaturas superiores a $450{ }^{\circ} \mathrm{C}$ son cristalinos, aumentando su cristalinidad al aumentar la temperatura. El refinamiento por el método de Rietveld de estos espectros demuestra que su estructura es la de un apatito carbonatado, similar a la parte mineral del hueso, donde los iones carbonato $\left(\mathrm{CO}_{3}^{2}\right)$ están sustituyendo parcialmente a los iones fosfato $\left(\mathrm{PO}_{4}^{3}\right)$ en la red cristalina.

El análisis por FT-IR (Fig. 2) corrobora el efectuado por XRD. Los espectros de las muestras producidas a las temperaturas más altas aparecen mucho más definidos que los de las obtenidas a las temperaturas mas bajas, indicando un aumento en su cristalinidad. Se pueden apreciar diferentes modos vibracionales de los iones $\mathrm{PO}_{4}^{3-}$ y $\mathrm{CO}_{3}^{2-}$, donde las posiciones de las bandas de $\mathrm{CO}_{3}^{2-}$ son típicas de apatitos carbonatados con sustitución parcial de $\mathrm{CO}_{3}^{2-}$ en el lugar del $\mathrm{PO}_{4}^{3-}$. También hay que señalar que la cantidad de $\mathrm{CO}_{3}^{2-}$ aumenta ligeramente con la temperatura.

La relación $\mathrm{Ca} / \mathrm{P}$ del recubrimiento depende tanto de la temperatura del substrato como de la presión de vapor de agua en la cámara de procesamiento. Al aumentar la temperatura aumenta la relación $\mathrm{Ca} / \mathrm{P}$, mientras que una presión mayor conduce a una menor $\mathrm{Ca} / \mathrm{P}$ (Fig. 3). Este comportamiento con la temperatura está de acuerdo con lo anteriormente señalado: si la cantidad de $\mathrm{CO}_{3}^{2-}$ aumenta es lógico que la $\mathrm{Ca} / \mathrm{P}$ aumente, al disminuir el contenido de $\mathrm{PO}_{4}^{3-}$.

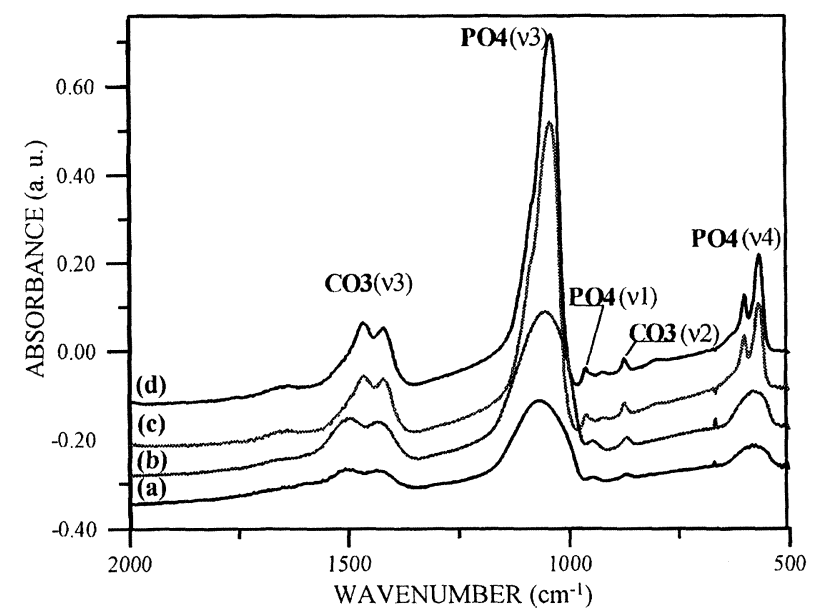

FIG. 2.- Espectros de FT-IR de los recubrimientos producidos por ablación láser a una presión de vapor de agua de 0,45 mbar y a diferentes temperaturas del substrato: a) $300, b) 430, c) 485$, y d) $525^{\circ} \mathrm{C}$.

FIG. 2.-FT-IR spectra of the coatings produced by laser ablation at 0.45 mbar of water vapour pressure and at different substrate temperatures: a) 300 , b) 430 , c) 485 , and d) $525^{\circ} \mathrm{C}$.

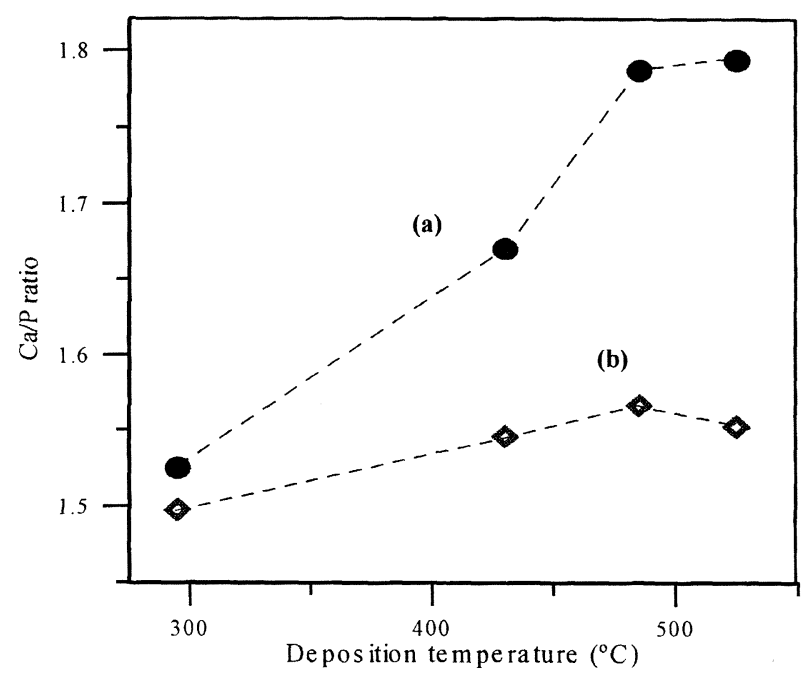

Fig. 3.- Relación $\mathrm{Ca} / \mathrm{P}$ vs temperatura de deposición de los recubrimientos producidos por ablación láser a presiones de vapor de agua de a) $0,3 \mathrm{y}$ b) 0,45 mbar.

FIG. 3.- Ca/P ratio vs deposition temperature of the coatings produced by laser ablation at a) 0.3 and b) 0.45 mbar of water vapour pressure.

El efecto de la presión de vapor de agua se observa más claramente en los espectros de XRD de los recubrimientos obtenidos a $485^{\circ} \mathrm{C}$ y a diferentes presiones (Fig. 4). A bajas presiones, los recubrimientos son amorfos debido a la incorporación excesiva de $\mathrm{CO}_{3}^{2-}$, tal como se muestra en el análisis por FT-IR de esas muestras (Fig. 5), que

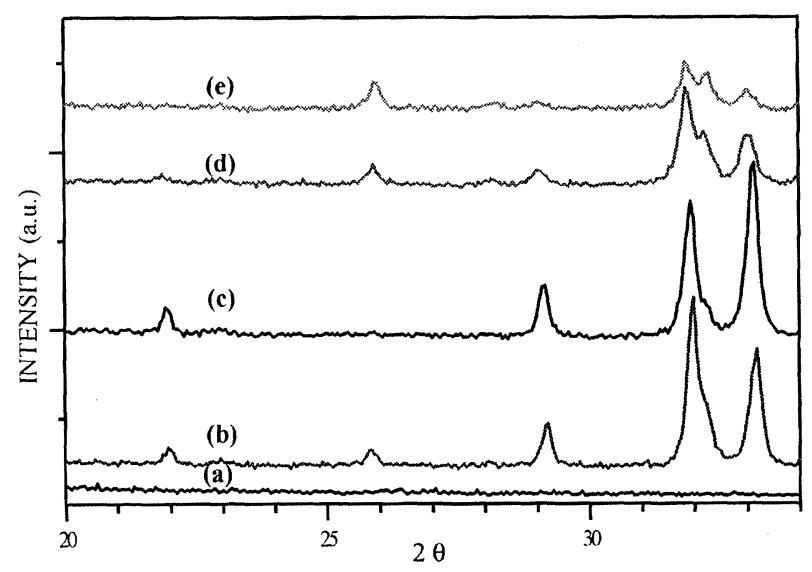

FIG. 4.- Espectros de XRD de los recubrimientos producidos por ablación láser a una temperatura del substrato de $485{ }^{\circ} \mathrm{C}$ y a diferentes presiones de vapor de agua: a) $0,15, b) 0,30, c) 0,45, d) 0,60$ and e) 0,80 mbar.

FIG. 4.-XRD spectra of the coatings produced by laser ablation at $485{ }^{\circ} \mathrm{C}$ of substrate temperature and at different water vapour pressures: a) 0.15, b) 0.30, c) 0.45 , d) 0.60 and e) 0.80 mbar. 


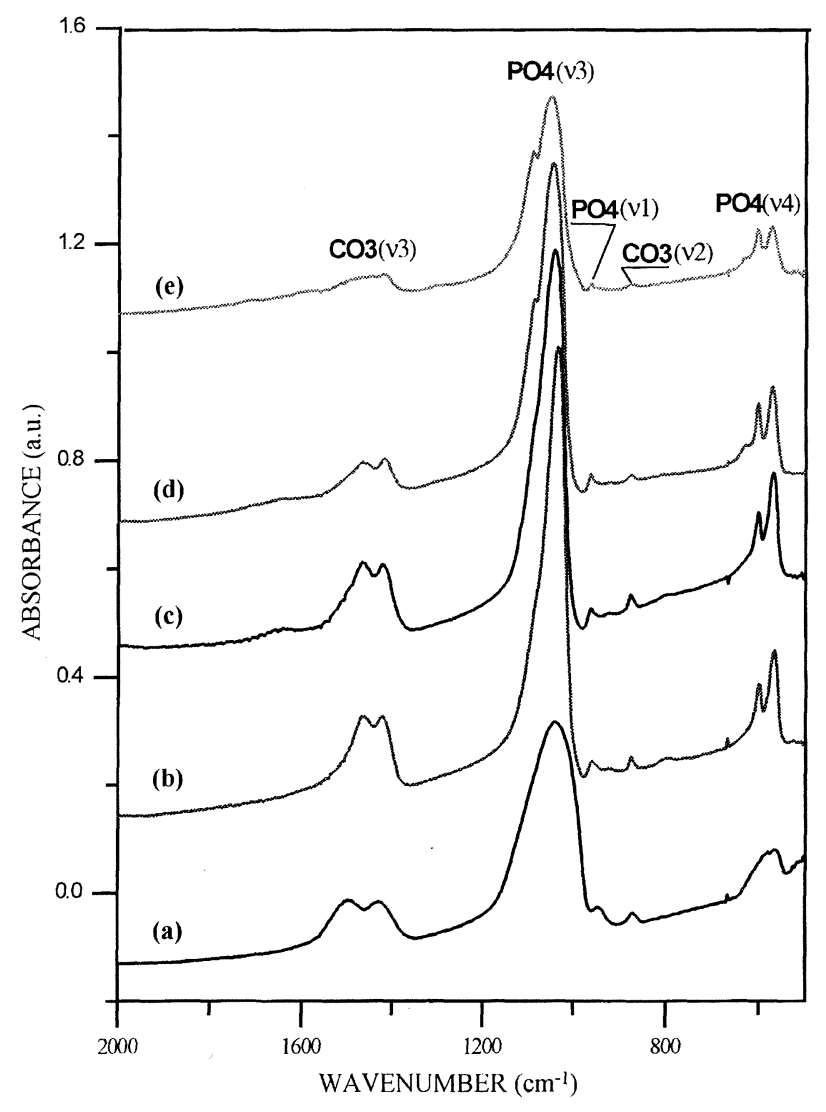

FIG. 5.- Espectros de FT-IR de los recubrimientos producidos por ablación láser a una temperatura del substrato de $485{ }^{\circ} \mathrm{C}$ y a diferentes presiones de vapor de agua: a) $0,15, b) 0,30, c) 0,45, d) 0,60 \mathrm{y}$ e) 0,80 mbar.

FIG. 5.- FT-IR spectra of the coatings produced by laser ablation at $485{ }^{\circ} \mathrm{C}$ of substrate temperature and at different water vapour pressures: a) 0.15,

b) 0.30 , c) 0.45 , d) 0.60 and e) 0.80 mbar.

destruye el orden de la estructura cristalina (14). En el rango 0,3-0,6 mbar, los recubrimientos son muy cristalinos, mientras que, a mayores presiones el contenido de $\mathrm{CO}_{3}^{2-}$ disminuye y la estructura de los recubrimientos corresponde a la de un apatito poco cristalino. Este ultimo efecto puede deberse a la incorporación de iones $\mathrm{Mg}^{2+}$ en el sitio del $\mathrm{Ca}^{2+}$ y de iones $\mathrm{HPO}_{4}^{2-}$ en la posición del $\mathrm{PO}_{4}^{3-}(15)$.

Todo este estudio demuestra que la técnica de ablación láser permite la producción de recubrimientos de fosfato cálcico de tipo apatito con diferentes propiedades físico-químicas. Este hecho puede ser de gran importancia desde el punto de vista de sus aplicaciones, ya que, dependiendo del tipo de hueso en el que se implante, una u otra composición del recubrimiento puede ser más favorable a la osteointegración del implante. Además, variando las condiciones de procesamiento durante la producción del recubrimiento, se puede obtener un gradiente en composición o cristalinidad, de modo que la parte externa se disuelva fácilmente por el ataque del medio fisiológico, dando lugar a un crecimiento del hueso en unión química con el implante, mientras que la parte metálica se encuentra protegida de dicho ataque por una parte interna difícil de disolver.

La figura $6 a$ muestra la morfología superficial del recubrimiento de fosfato cálcico producido mediante la técnica de ablación láser. Mediante medidas de XRD se ha determinado que dicho recubrimiento es altamente cristalino. Toda la superficie de titanio está recubierta por una red de pequeños cristales de apatito de forma poligonal de tamaños comprendidos entre 100 y $500 \mathrm{~nm}$.

Las figuras $6 b$ - $d$ muestran diferentes vistas (a diferentes aumentos) de secciones transversales del recubrimiento SEM. Las fotomicrografías corresponden a cilindros de titanio que han sido recubiertos para las pruebas biológicas que se llevan a cabo actualmente. El recubrimiento es denso y homogéneo y no se detecta grieta alguna en toda su longitud. La resina acrílica usada en la preparación de la muestra está ligeramente separada de la superficie del recubrimiento. A pesar del desprendimiento de la resina por efecto del haz de electrones del SEM, el recubrimiento permanece firmemente sujeto al substrato de titanio. Esto constituye una evidencia de la buena adhesión entre el recubrimiento y el substrato.

Los resultados de las pruebas de tracción confirman las observaciones hechas por SEM. La resistencia a la tracción supera las $58 \mathrm{MPa}$, produciéndose la fractura por el adhesivo en todos los casos, sin desprendimiento del recubrimiento. Este es un resultado muy bueno, teniendo en cuenta el hecho de que los substratos de titanio no se granallaron como siempre es necesario en el caso de los recubrimientos comerciales producidos por plasma-spray.

Las pruebas de biocompatibilidad realizadas in vitro indicaron que los recubrimientos seleccionados no resultaron ser citotóxicos y que la proliferación de células de tipo osteoblasto fue mejor que en el caso de los recubrimientos producidos por plasma-spray utilizados como controles.

\section{CONCLUSIONES}

La técnica de ablación láser permite producir recubrimientos finos de fosfato cálcico de gran adherencia. Sus propiedades físico-químicas se pueden seleccionar fácilmente escogiendo adecuadamente los parámetros de procesamiento. La ablación láser parece una buena candidata para solucionar los principales problemas de las técnicas de recubrimiento comerciales. 

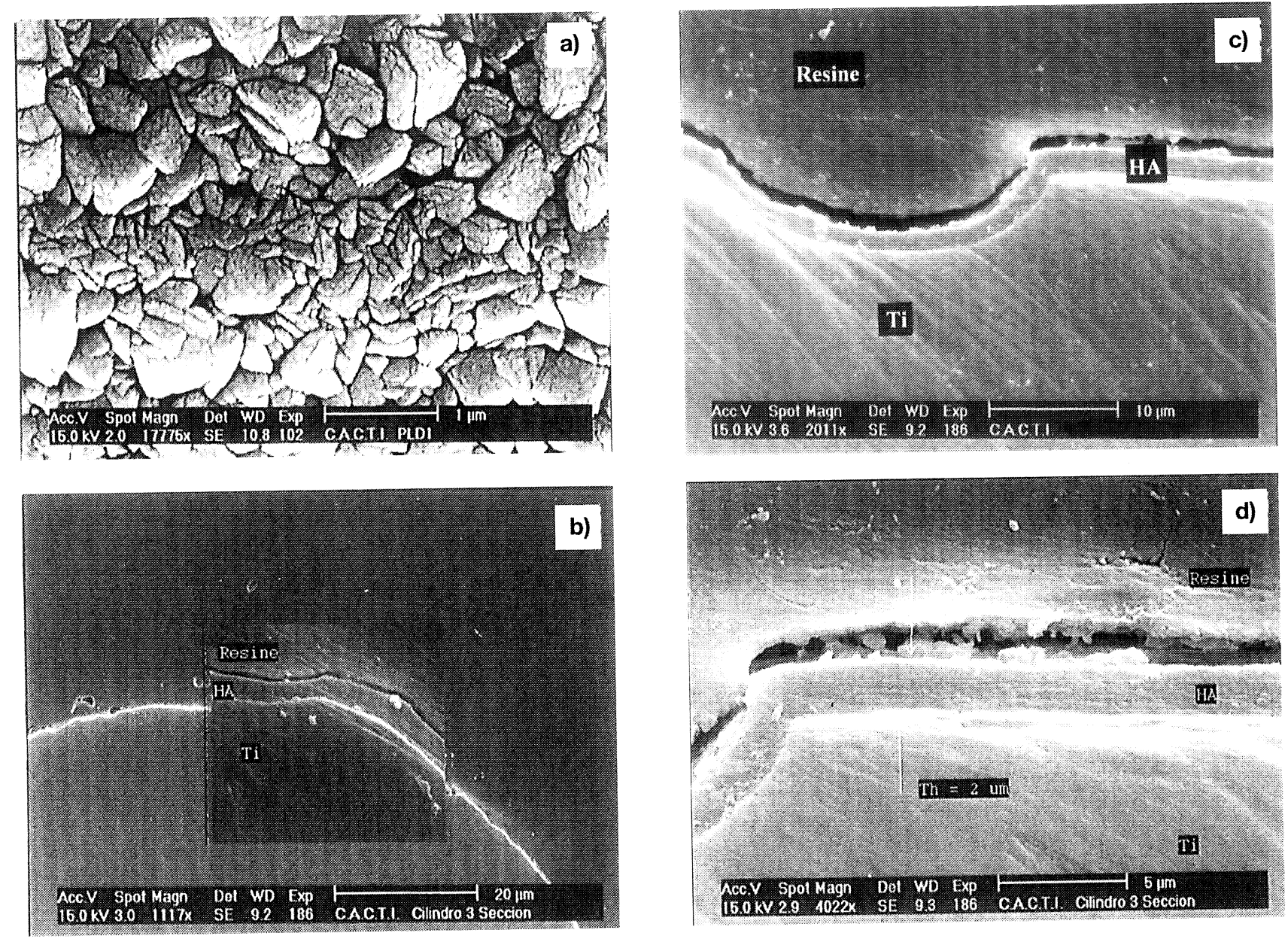

FIG. 6.- Fotomicrografías SEM que muestran la superficie y la sección transversal (a diferentes ampliaciones) de un cilindro recubierto.

FIG. 6.- SEM micrographs showing the surface and cross section (at different magnifications) of a coated cylinder.

\section{Agradecimiento}

Los autores quieren agradecer a los Profs. W. Bonfield, F. Driessens y J. Arends su apoyo y enriquecedoras discusiones. Se agradece profundamente la asistencia técnica del C.A.C.T.I. (Universidad de Vigo). Gracias en especial a CIDA s.a.l. (Barcelona) por las pruebas biológicas y a INASMET (San Sebastián) por las medidas mecánicas. Este trabajo ha sido parcialmente subvencionado por la Unión Europea (contrato CRAFT BRE2.CT94. 1533), la CICYT (MAT93-0271), el MINER (Programa PATI 665/95) y la Xunta de Galicia (INFRA94-58).

\section{REFERENCIAS}

(1) LeGeros, R.Z. Adv.Dent.Res., 2, 1988: 164.

(2) HenCH, L.L. and Wilson, J. An Introduction to Bioceramics, World Scientific, Singapore, 1993: 1-24.

(3) Kay, J.F. Dent. Clin. North. Am. 36, 1992: 1.

(4) Filiaggi, M.J., Pilliar, R.M. y CoOmbs, N.A. J. Biomed. Mater. Res. 27, 1993: 191.
(5) Cheang, P. y Khor, K.A. J. Mater. Proc. Technol., 48, 1995: 429.

(6) Shirkhanzadeh, M., Azadegan, M., Stack, V. y SCHREYER, S. Mater. Letters, 18, 1994: 211.

(7) Wolke, J.G.C., Van Dijk, K., Schaeken, H.G., De Groot, K. y Jansen, J.A. J. Biomed. Mater. Res.28, 1994: 1477.

(8) Chen, T.S. y LaCeField, W.R. J. Mater. Res. 9, 1994: 1.284.

(9) Cotell, C.M., Chrisey, D.B., Grabowski, K.S., Sprague, J.A. y GossetT, C.R. J. Appl. Biomat. 3, 1992: 87.

(10) Baeri, P., Torrisi, L., Marino, N. y Foti, G. Appl. Surf. Sci. 54, 1992: 210.

(11) Sardin, G., Varela, M. y Morenza, J.L. Hydroxyapatite and related materials, P.W. Brown \& B. Constanz, CRC Press, Boca Raton, 1994: 225

(12) Mayor, B., Arias, J., Chiussi, S., Garcia, F., Pou, J., Leon, B. y Pérez-Amor, M. Thin Solid Films, (Pendiente de publicación).

(13) Leon, B., Pou, J., Arias, J.L., Garcia-Sanz, F.J., Mayor, M.B., Gonzalez, P. y PÉrez-Amor, M. Pat. Española P9602439, 1997.

(14) LeGeros, R.Z., Trautz, O.R., LeGeros, J.P. y Klein, E. Bull. Soc. Chim. Fr., Núm. especial 1968: 1.712.

(15) Arias, J.L., Mayor, M.B., Garcia-Sanz, F.J., Pou, J., Leon, B., Pérez-Amor, M. y Knowles, J.C. J. Mater. Sci. Mater Med. 8, 1997: 873-876. 